\title{
Rapamycin targets several pathophysiological features of immune-mediated bone marrow failure in murine models
}

\author{
Wendy W. Weston, ${ }^{1,2}$ Vesna Jurecic ${ }^{1}$ and Roland Jurecic ${ }^{1}$ \\ ${ }^{1}$ Department of Microbiology and Immunology, Miller School of Medicine, University of Miami, FL and ${ }^{2}$ Cell Therapy Institute, College of \\ Medicine, Nova Southeastern University, Fort Lauderdale, FL, USA
}

E-mail: rjurecic@med.miami.edu

doi:10.3324/haematol.2017.175497

$I^{a}$ this issue of Haematologica, Feng et al. ${ }^{1}$ compare the efficacy of treatment with cyclosporine A (CsA) and rapamycin to ameliorate pancytopenia, improve bone marrow (BM) cellularity, and extend survival in murine models of immune-mediated aplastic anemia (AA). Interestingly, while the efficacy of $\mathrm{CsA}$ and rapamycin to attenuate immune-mediated bone marrow failure (BMF) in murine AA models is similar, CsA and rapamycin achieve their effects through different mechanisms. ${ }^{1}$

Immune-mediated aplastic anemia (AA) is an acquired form of BMF and is characterized by an abnormally low number of BM cells (hypoplasia) and severe reduction in blood cells (pancytopenia), which in the severe form of AA (SAA) can be life-threatening. The immune and hematologic pathophysiology of AA is quite complex and includes: a) development and oligoclonal expansion of autoreactive $T$ cells, including $\mathrm{CD}^{+}$cytotoxic $\mathrm{T}$ cells, CD4 ${ }^{+} \mathrm{Th} 1$ cells, and Th17 cells; b) effector T-cell-mediated apoptosis and depletion of hematopoietic stem and progenitor cells (HSPCs) and mature blood cells, leading to BM hypoplasia and pancytopenia; c) production of proinflammatory cytokines (e.g. TNF $\alpha$ and IFN $\gamma$ ); d) severe reduction and functional impairment of immunosuppressive regulatory $\mathrm{T}$ cells (Tregs); and e) karyotype abnormalities, genomic instability, and somatic mutations in different myeloid cancer-associated genes that positively and negatively correlate with response to immunosuppresive therapy (IST) and risk of development of myelodysplasia and acute myeloid leukemia (AML) ${ }^{2-6}$

Current standard treatments for AA include IST with horse anti-thymocyte globulin (ATG) and cyclosporine A (CsA), or allogeneic HLA-matched sibling or well-matched unrelated donor BM transplant. While IST is effective in 60$70 \%$ of AA patients, a significant proportion of patients who responded to IST undergo relapse after CsA withdrawal or are refractory to IST. Moreover, IST is not effective in treating refractory and relapsed $\mathrm{AA}^{7.12}$

Recently, combined application of eltrombopag, a thrombopoietin mimetic, and standard IST has proven to be very effective in treating patients with refractory and severe AA. However, relapse and clonal evolution remain important post-therapy concerns..$^{13,14}$

Different murine models were developed to study the etiology and pathophysiology of AA, and the MHC partially mismatched lymphocyte infusion models, based on alloantigen recognition, are the best characterized and most relevant pre-clinical AA models. The induced AA in these models exhibits many of the clinical and pathological features of acquired AA in patients, and can be modulated using IST and Treg cell therapies. These models provided important insights into the cellular and molecular immune effectors implicated in AA, and are a powerful and relevant in vivo sys- tem for testing new drugs and therapeutic approaches for treating SAA. ${ }^{15,16}$

The AA in lymphocyte infusion models is induced by infusing parental lymph node cells (LNCs) from $\mathrm{H}_{2}{ }^{\mathrm{b}}$ C57BL/6 mice into MHC partially mismatched non-irradiated or sublethally irradiated F1 hybrid H2 ${ }^{\text {b/d }}$ B6D2F1 (C57BL6 $\mathrm{x} \mathrm{DBA} / 2 \mathrm{~J}$ ) or CByB6F1 (C57BL6 $\mathrm{x} \mathrm{BALB} / \mathrm{c}$ ) recipients. Among mismatched minor-H antigens, $\mathrm{H} 60$ contributes the most to AA development in the C.B10 mouse AA model, which is generated by infusion of LNCs from BL6 mice into C.B10 mice which are pre-conditioned with 5 Gy of sublethal total body irradiation (TBI). ${ }^{15,16}$

Feng et al. have shown that treatment of AA mice with rapamycin for 12 days and treatment with CsA for nine days resulted in similar and statistically significant improvements in BM cellularity, number of white blood cells (WBCs) and platelets (PLTs), and 100-day survival in comparison to untreated AA mice and control mice that received 5 Gy TBI. Temporal studies of recovery of complete blood counts (CBCs) and BM cellularity in rapamycin-treated mice and TBI control mice revealed a similar degree of recovery at days (d)28, 42 and 100, except for a delayed WBC recovery in rapamycin-treated mice. ${ }^{1}$

Importantly, delayed treatment with rapamycin was also effective in decreasing pancytopenia and BMF in mice with ongoing AA, with a better response from treatment initiated at $\mathrm{d} 5$ versus $\mathrm{d} 7$ after $\mathrm{LN}$ cell infusion. Moreover, the therapeutic effects of a 5-day delay of rapamycin treatment lasted for ten weeks in this experiment, albeit with a significantly slower recovery of WBCs.

Subsequent experiments have demonstrated that both $\mathrm{CsA}$ and rapamycin rescued mice from BM failure by suppressing $\mathrm{CD}^{+}$and $\mathrm{CD}^{+}$T-cell infiltration in the $\mathrm{BM}$. However, treatment of AA mice with rapamycin led to an increase in functional regulatory $\mathrm{T}$ cells in the BM, lymph node and spleen in comparison to mice treated with CsA and untreated AA mice. Furthermore, rapamycin more efficiently eradicates $\mathrm{CD}^{+}$effector $\mathrm{T}$ cells in a CByB6F1 AA model and antigen-specific $\mathrm{CD}^{+}$effector $\mathrm{T}$ cells in a minor histocompatibility antigen $\mathrm{H} 60$ mismatched AA model. Additional in vitro and in vivo experiments have revealed that rapamycin treatment is more efficient in reducing memorylike and effector T cells than CsA treatment. ${ }^{1}$

Transcriptome analyses of $\mathrm{BM} \mathrm{CD}^{+}$and $\mathrm{CD}^{+} \mathrm{T}$ cells from $\mathrm{BMF}$ mice with or without rapamycin or CsA treatment have discovered important differences in the transcription profile of effector molecules important for immune activity of cells, indicating that rapamycin and CsA exert their immuno-modulating effects through different molecular pathways. Furthermore, the analysis of cytokine secretory profiles of $\mathrm{T}$ cells from rapamycin and CsA-treated mice 
revealed significant differences in the effects of rapamycin and CsA on cytokines related to Th1 and Th2 immune responses. ${ }^{1}$ These important mechanistic findings warrant further molecular and functional studies to uncover the full spectra of molecular and physiological mechanisms of immunosuppression through which rapamycin and CsA ameliorate BMF.

Through significant depletion of effector $\mathrm{T}$ cells and increase in Treg cells, treatment with rapamycin resulted in improved BM cellularity, significantly lower pancytopenia, and significantly increased numbers of HSPCs in the BM and long-term survival of mice with AA. Thus, similar to other experimental anti-inflammatory and immunosuppressive approaches, ${ }^{16,17}$ treatment with rapamycin simultaneously and efficiently targets several pathophysiological features of $\mathrm{AA}$ in murine models.

The analysis of HSPC populations in the BM of control, TBI, untreated, CsA-treated and rapamycin-treated mice on $\mathrm{d} 13$, has revealed that rapamycin treatment resulted in a 2-3-fold increase in the frequency and numbers of c-

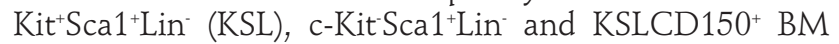
cells, which greatly surpass the numbers observed in control mice. Interestingly, in contrast to control, TBI, untreated and CsA-treated mice, c-Kit ${ }^{+}$Sca ${ }^{+}{ }^{+}$Lin $(\mathrm{KSL})$ and c-Kit-Sca1+Lin cells from rapamycin-treated mice exhibited significantly increased numbers of cells with high expression of Sca-1 marker. It is well established that inflammatory conditions (radiation, chemotherapy, infections) and inflammatory cytokines such as IFNs and TNF $\alpha$ increase Sca-1 expression on HSPCs. ${ }^{18,19}$

Thus, it is unclear at this point what the cause of significant Sca-1 upregulation is, since the cytokine profiling of plasma from rapamycin-treated mice on $\mathrm{d} 13$ has shown that rapamycin significantly down-regulated both IFN $\gamma$ and TNF $\alpha$. It would be very interesting to analyze c$\mathrm{Kit}^{+} \mathrm{Sca}{ }^{+}{ }^{+} \mathrm{Lin}-(\mathrm{KSL}), \mathrm{c}-\mathrm{Kit} \mathrm{Sca}^{+}{ }^{+} \mathrm{Lin}^{-}$and KSLCD150+ BM cells in rapamycin-treated mice at later time points during their long-term survival.

Analysis of effects of CsA and rapamycin on mTOR and NFAT signaling pathways suggests that CsA suppresses immune activity by interfering with the NFAT1 signaling pathway, whereas rapamycin promotes differentiation of Th2 effector lineages and suppresses proinflammatory Th1 and Th17 T cell lineages by modulating mTOR activity.

In conclusion, the study by Feng et al. demonstrates that, similar to treatment with standard dose of CsA, rapamycin effectively and reproducibly attenuated immune-mediated BM failure in mouse models of AA. ${ }^{1}$

Although treatment of AA patients with standard IST and rapamycin in a recent clinical trial was not more effi- cient than standard IST, ${ }^{20}$ due to its immunosuppressive activity and tolerogenic role in organ transplantation, rapamycin has clinically relevant potential and will be tested in an upcoming phase II clinical trial as a prophylactic treatment of AA patients at high risk of relapse after withdrawal of CsA treatment.

\section{References}

1. Feng X, Lin Z, Sun W, Hollinger MK, et al. Rapamycin is highly effective in murine models of immune-mediated bone marrow failure. Haematologica. 2017;102(10):1691-1703.

2. Young NS. Current concepts in the pathophysiology and treatment of aplastic anemia. Hematology Am Soc Hematol Educ Program. 2013;2013:76-81.

3. Zeng Y, Katsanis E. The complex pathophysiology of acquired aplastic anaemia. Clin Exp Immunol. 2015;180(3):361-370.

4. Boddu PC, Kadia TM. Updates on the pathophysiology and treatment of aplastic anemia: a comprehensive review. Expert Rev Hematol. 2017;10(5):433-448.

5. Ogawa S. Clonal hematopoiesis in acquired aplastic anemia. Blood. 2016;128(3):337-347

6. Stanley N, Olson TS, Babushok DV. Recent advances in understanding clonal haematopoiesis in aplastic anaemia. Br J Haematol. 2017;177(4):509-525.

7. Georges GE, Storb R. Hematopoietic stem cell transplantation for acquired aplastic anemia. Curr Opin Hematol. 2016;23(6):495-500.

8. Risitano AM. Immunosuppressive therapies in the management of acquired immune-mediated marrow failures. Curr Opin Hematol. 2012;19(1):3-13.

9. Marsh JC, Kulasekararaj AG. Management of the refractory aplastic anemia patient: what are the options? Hematology Am Soc Hematol Educ Program. 2013;2013:87-94.

10. Schrezenmeier H, Körper S, Höchsmann B. Immunosuppressive therapy for transplant-ineligible aplastic anemia patients. Expert Rev Hematol. 2015;8(1):89-99.

11. Dietz AC, Lucchini G, Samarasinghe S, et al. Evolving hematopoietic stem cell transplantation strategies in severe aplastic anemia. Curr Opin Pediatr. 2016;28(1):3-11.

12. Bacigalupo A, Giammarco S, Sica S. Bone marrow transplantation versus immunosuppressive therapy in patients with acquired severe aplastic anemia. Int J Hematol. 2016;104(2):168-174.

13. Townsley DM, Scheinberg P, Winkler T, et al. Eltrombopag Added to Standard Immunosuppression for Aplastic Anemia. N Engl J Med. 2017;376(16):1540-1550.

14. Lum SH, Grainger JD. Eltrombopag for the treatment of aplastic anemia: current perspectives. Drug Des Devel Ther. 2016;10:2833-2843.

15. Chen J. Animal models for acquired bone marrow failure syndromes. Clin Med Res. 2005;3(2):102-108.

16. Scheinberg P, Chen J. Aplastic anemia: what have we learned from animal models and from the clinic. Semin Hematol. 2013;50(2):156164.

17. Weston W, Gupta V, Adkins R, et al. New therapeutic approaches for protecting hematopoietic stem cells in aplastic anemia. Immunol Res. 2013;57(1-3):34-43

18. King KY, Goodell MA. Inflammatory modulation of HSCs: viewing the HSC as a foundation for the immune response. Nat Rev Immunol. 2011:11(10):685-692.

19. Schuettpelz LG, Link DC. Regulation of hematopoietic stem cell activity by inflammation. Front Immunol. 2013;4:204.

20. Scheinberg $\mathrm{P}, \mathrm{Wu} \mathrm{CO}$, Nunez $\mathrm{O}$, et al. Treatment of severe aplastic anemia with a combination of horse antithymocyte globulin and cyclosporine, with or without sirolimus: a prospective randomized study. Haematologica. 2009;94(3):348-354. 